\title{
Modelos de predição da redução do consumo energético em edifícios que utilizam a iluminação natural através de regressão linear multivariada e redes neurais artificiais
}

\author{
Models for predicting the reduction of energy \\ consumption in buildings using daylighting through a \\ multivariate linear regression and artificial neural \\ networks
}

Raphaela Walger da Fonseca Laboratório de Conforto Ambiental, Departamento de Arquitetura e Urbanismo Universidade Federal de Santa Catarina Caixa-Postal 476
Trindade Florianopolis - SC - Brasil CEP 88040-900 Tel.: (48) 3721-4974 E-mail: raphawf@gmail.com

Evelise Leite Didoné Building Science Group Karlsruhe Institute of Technology

Englerstrasse 7 D-76131 Karlsruhe - Germany Tel.: +49 (0) 72160842178 E-mail: evelise.didone@kit.edu

Fernando Oscar Ruttkay Pereira Departamento de Arquitetura e Urbanismo, Centro Tecnológico Universidade Federal de Santa Catarina Caixa-Postal 476 Trindade Florianopolis - SC - Brasil CEP 88040-900 Tel.: (48) 3721-7080 E-mail: feco@arq.ufsc.br

Recebido em 10/10/11 Aceito em 06/12/11

\section{Raphaela Walger da Fonseca \\ Evelise Leite Didoné \\ Fernando Oscar Ruttkay Pereira}

\section{Resumo}

M

uitos estudos já comprovaram a preferência dos usuários pela luz natural como fonte de luz em edificações. Além de benefícios à saúde atribuídos a sua influência no ritmo circadiano humano, a elevada qualidade na reprodução de cor, entre outros aspectos, a luz natural apresenta reconhecido potencial de economia de energia quando substitui ou complementa a iluminação artificial. Outro fator a ser considerado é que a maior disponibilidade de iluminação natural coincide com o horário comercial de funcionamento de edificações de escritório. Neste contexto, o objetivo deste trabalho é demonstrar um comparativo entre dois modelos de aproximação de funções utilizadas para representar o potencial de economia de energia través do uso da iluminação natural em escritórios. São eles: Regressão Linear Multivariada e Regressão Não Linear Multivariada, também conhecida como Rede Neural Artificial (RNA). Os resultados mostraram que as RNAs são mais adequadas para esse tipo de problema devido a sua grande habilidade de aprender, o que permite melhor capacidade de generalizar os dados em relação à Regressão Linear Multivariada.

Palavras-chave: Iluminação natural. Eficiência energética. Redes neurais artificiais.

\section{Abstract}

Many studies have confirmed users' preference for daylight as a light source in buildings. In addition to the health benefits attributed to its influence on human circadian rhythms, high quality colour reproduction and other aspects, daylight has a known potential for energy savings when replacing or supplementing artificial lighting. Another factor to be considered is that the availability of daylight coincides with the working hours of commercial office buildings. In this context, the objective of this paper is to draw a comparison between two types of approximation methods used to estimate the potential energy savings through the use of daylight in office buildings. These approximation methods models are: Linear and Nonlinear Multivariate Regression, also known as Artificial Neural Network (ANN). The results show that ANNs are particularly suitable for this type of problem due to their learning aptitude, which allows significantly better extrapolation of the learning data than Multivariate Linear Regression.

Keywords: Daylight. Energy efficiency. Neural networks. 


\section{Introdução}

Existe grande número de pesquisas que mostram a preferência pela luz natural como fonte de luz em edifícios. Esse fato deve-se a uma série de fatores relacionados com o cumprimento das necessidades biológicas humanas, além do potencial de economia de energia mediante a substituição da iluminação artificial pela iluminação natural (MAYHOUB; CARTER, 2011).

Reinhart e Wienold (2011) propõem uma definição híbrida para a iluminação natural que condiz com o que se espera de projetos que tiram partido dessa fonte de luz nos dias de hoje. Um espaço que é essencialmente iluminado pela luz do dia deve também combinar elevada satisfação dos ocupantes considerando conforto visual e térmico com baixo consumo energético para iluminação artificial, aquecimento e resfriamento.

Algumas pesquisas no cenário nacional, como Souza (2003), Carlo, Pereira e Lamberts (2004) e Didoné (2009), já contribuíram para caracterizar o potencial de economia de energia através da iluminação natural em edificações não residenciais. Entretanto, nenhuma delas considerou o uso de Regressão Não Linear Multivariada para a aproximação de funções. As técnicas de Regressão Não Linear Multivariada através de Redes Neurais Artificiais (RNAs) têm sido utilizadas com boa resposta para modelagens em várias áreas de pesquisa (KAZANASMAZ; GÜNAYDIN; BINOL, 2009). A motivação para a utilização dessa técnica é sua boa resposta para modelos não lineares. Para tornar mais efetivo o uso das RNAs, depois de treinada, a rede pode ser convertida em uma ferramenta em formato de planilha, o que facilita sua aplicação para a avaliação de novos casos (BOCANEGRA, 2002).

No sentido de avançar nesse tipo de avaliação, este trabalho busca, por meio de simulação computacional e uso de técnicas de RNAs, definir uma metodologia utilizando regressão não linear para representar funções de problemas concernentes à eficiência energética sob a ótica do aproveitamento da iluminação natural. Para tal, é feita uma comparação entre resultados obtidos através de RNA e de Regressão Linear Multivariada.

\section{Revisão bibliográfica \\ Eficiência energética e iluminação natural}

Nos últimos anos, quando se começou a falar mais sistematicamente sobre conservação e racionalização do uso da energia elétrica nos edifícios, o aspecto da integração entre luz natural e artificial passou a assumir importância cada vez maior. A eficiência energética de um sistema de iluminação artificial também pode ser vinculada à redução do desperdício de energia de um sistema acionado quando o ambiente está desocupado.

Assaf e Pereira (2003) trazem os resultados de uma pesquisa sobre o desperdício da iluminação ligada em espaços desocupados atingindo um valor médio de $7 \%$ para residências unifamiliares e de $23 \%$ para edifícios de administração estatais. Li e Tsang (2008), em pesquisa sobre o potencial de economia de energia, obtiveram que a região perimetral de plantas livres de escritórios pode ter $50 \%$ de suas horas de ocupação atendidas pela luz natural. Com isso, a redução no consumo geral dos edifícios pode alcançar de $20 \%$ a $25 \%$.

Projetos desenvolvidos objetivando o aproveitamento da luz natural chegam a alcançar a iluminância requisitada nos interiores em $80 \%$ a $90 \%$ das horas diurnas do ano, economizando consideráveis quantidades de energia elétrica (VIANNA; GONÇALVES, 2001). Souza (1995) analisou o comportamento energético de prédios de escritórios mediante simulações computacionais e concluiu que o aproveitamento da iluminação natural poderia reduzir o consumo total de energia elétrica em até $35 \%$.

\section{Regressão Linear Multivariada na avaliação da eficiência energética}

A Regressão Linear Multivariada objetiva aumentar o conhecimento sobre a relação entre diversas variáveis independentes e uma variável dependente. As equações baseiam-se em um universo amostrado e permitem que qualquer número seja inserido, como numa regressão matemática; possui algumas limitações, como uma equação estatística que descreve uma realidade (CARLO; GHISI; LAMBERTS, 2003).

Em diversas áreas do conhecimento procedimentos de Regressão Multivariada são amplamente utilizados em análise de pesquisas. As equações podem ser usadas para muitas finalidades. Uma delas é a análise paramétrica sobre os parâmetros de construção, como na avaliação da envoltória com diferentes tamanhos de janelas, tipos de vidros, proteção solar, etc. Outra seria a aplicação pelos decisores políticos para configurar os níveis de eficiência energética no setor da construção civil (WESTPHAL; LAMBERTS, 2007).

$\mathrm{Na}$ área de eficiência energética em edificações, pesquisas relacionadas à elaboração de códigos e 
normas fazem uso do modelo de Regressão Linear Multivariada em suas análises. Como exemplo pode-se citar o trabalho de Signor (1999), que com mais de 7.000 simulações paramétricas desenvolveu uma correlação para estimar o consumo de energia elétrica em edificações para 14 climas brasileiros. Essas equações foram um dos pontos de partida para o desenvolvimento de padrões de eficiência energética para edifícios comerciais no Brasil.

A primeira lei de eficiência energética do Brasil foi aprovada em 2001, quando o Governo Federal publicou a Lei $\mathrm{n}^{\circ} 10.295$ (BRASIL, 2001), sobre o uso racional da energia, indicando a necessidade da criação de indicadores técnicos referenciais do consumo e da eficiência de edificações. Nesse contexto, foi submetido para a cidade de Salvador um estudo para incluir parâmetros de eficiência energética relacionados ao envelope da edificação em seu código de obras. O estudo com uso de simulação computacional permitiu desenvolver equações de Regressão Linear Multivariada para a análise dos parâmetros da envoltória de edificações (CARLO; GHISI; LAMBERTS, 2003).

Dando continuidade, Carlo (2008) criou um indicador de eficiência energética para o envoltório das edificações mediante o desenvolvimento de uma equação de Regressão Linear Multivariada. As equações também foram obtidas a partir de simulação computacional termoenergética de protótipos de edifícios no programa EnergyPlus. As mesmas equações foram utilizadas no desenvolvimento do método prescritivo do Regulamento Técnico da Qualidade do Nível de Eficiência Energética (RTQ-C), publicado no Brasil em fevereiro de 2009, como parte do Programa Brasileiro de Etiquetagem do Instituto Nacional de Metrologia, Qualidade e Tecnologia (Inmetro).

Essas pesquisas comprovam que o modelo de Regressão Linear Multivariada para análise do consumo de energia elétrica dos edifícios pode ser usado como uma forma alternativa e rápida para analisar o desempenho energético e o uso da luz natural de acordo com os parâmetros selecionados.

\section{Redes Neurais Artificiais}

As redes neurais artificiais (RNAs) foram inspiradas na estrutura biológica do cérebro humano, fazendo-se uma analogia com o funcionamento dos neurônios. As RNAs têm por objetivo fornecer subsídios para que a ferramenta computacional consiga, com base em um conjunto de simulações conhecidas, estender tais informações para determinada situação proposta (COSTA, 2001).
De acordo com Bocanegra (2002), as RNAs apresentam elevado potencial para a solução de problemas na área de engenharia, não apenas pela forma como esses problemas são representados internamente pela rede, mas também pelos resultados gerados por ela. Isso porque as RNAs vêm demonstrando desempenho superior a modelos tradicionais, como as análises de regressão, por exemplo.

Haykin (2001) define como benefícios das redes neurais, primeiro, sua estrutura paralelamente distribuída e, depois, sua habilidade de aprender e generalizar, o que quer dizer que a RNA produz resultados adequados para dados de entrada que não pertenceram ao treinamento.

Alguns autores já utilizaram as RNAs na avaliação de iluminação natural. Pizarro e Souza (2007) avaliaram a importância e as relações entre as variáveis do conforto luminoso em escolas públicas. As autoras trabalharam com medições de iluminâncias e utilizaram as RNAs como ferramenta de modelagem das relações entre as variáveis levantadas.

Kazanasmaz, Günaydin e Binol (2009) utilizaram as RNAs para a formulação de um modelo de predição de iluminação natural desenvolvido para determinar iluminâncias em edifícios de escritório. Foram utilizadas as iluminâncias medidas em campo, como no estudo anterior. A arquitetura da RNA consistiu em um modelo com três níveis e apenas uma variável de saída. Dos 100 modelos, 80 foram usados para o treinamento (fase de aprendizado) da RNA e 20 foram deixados para a fase de testes. O desempenho do modelo foi testado usando-se o percentual de erro da iluminância, obtendo uma aproximação de 98\% com os dados reais. A principal conclusão dos autores foi que modelos de RNA podem ser usados como ferramenta de projeto, já que, nesse caso, apresentaram alta capacidade de predição de iluminâncias, de forma a oferecer respostas de forma rápida.

\section{Neurônio artificial}

Haykin (2001) define o neurônio artificial como uma unidade de processamento matematicamente simples, pois recebe uma ou mais entradas, que, por sua vez, têm um peso associado e as transforma em saídas. Pode-se complementar ainda que o neurônio é composto de uma função soma, ${ }^{1}$ responsável pelo cálculo da entrada efetiva para o

\footnotetext{
${ }^{1}$ Função soma: soma os sinais de entrada, ponderados pelas respectivas sinapses (pesos) do neurônio.
} 
neurônio, e uma função ativação ${ }^{2}$ ou função de transferência.

O conhecimento da rede é representado pelos pesos, que determinam a importância que cada entrada possui. A influência que cada entrada terá na saída será tanto quanto maior e mais estimulada for uma conexão sináptica ${ }^{3}$, pois mais frequente será a atualização do respectivo peso.

\section{Rede multilayer perceptron}

Esses tipos de redes constituem os modelos RNA mais difundidos no momento. Consistem em um conjunto de unidades sensoriais, formando uma camada de entrada, uma ou mais camadas intermediárias de unidades ocultas e uma camada de saída. A propagação dos sinais de entrada acontece de camada a camada na direção positiva (da entrada para a saída) (BOCANEGRA, 2002).

Cada unidade da camada intermediária funciona como um perceptron ${ }^{4}$. O tipo de ativação escolhido dá-se de forma a suavizar a atualização dos pesos, evitando alterações bruscas (COSTA, 2001).

As redes multilayer perceptron (MLP) têm sido aplicadas de maneira satisfatória para problemas envolvendo alto grau de não linearidade. O treinamento aplicado às MLP é o do tipo supervisionado. $\mathrm{O}$ algoritmo utilizado é o chamado retropropagação do erro (error backpropagation), baseado numa regra de aprendizagem que busca corrigir o erro durante a fase de treinamento (HAYKIN, 2001).

\section{Processo de aprendizagem}

A habilidade de aprender é uma das principais propriedades de uma rede neural. $\mathrm{O}$ treinamento de uma rede neural tem por objetivo produzir um conjunto de saídas desejadas ou mesmo consistentes mediante a aplicação de determinado conjunto de entradas. Cada um desses conjuntos (de entrada ou saída) denomina-se vetor. Realizase o treinamento através da aplicação sequencial de vetores de entrada e, algumas vezes também, de saída, enquanto se ajustam os pesos da rede

\footnotetext{
${ }^{2}$ Função de ativação: restringe a amplitude de saída de um neurônio. Limita o intervalo permissível de amplitude do sinal de saída a um valor finito, normalizado como o intervalo unitário fechado $[0,1]$ ou $[-1,1]$. É a função responsável pela propagação da saída de um neurônio para os demais neurônios.

${ }^{3}$ Conexão sináptica: as sinapses são unidades estruturais e funcionais elementares que intermedeiam as interações entre os neurônios. Na organização neural, uma sinapse é uma conexão simples que pode impor ao neurônio receptivo excitação ou inibição, mas não ambas (HAYKIN, 2001).

${ }^{4}$ Perceptron: composto de um neurônio, uma função limiar e aprendizado supervisionado. Sua arquitetura consiste na entrada e numa camada de saída.
}

conforme um procedimento de treinamento escolhido previamente. Durante o processo de treinamento os pesos vão gradualmente convergindo para determinados valores, até que os vetores de entrada produzam as saídas desejadas (BOCANEGRA, 2002).

Os procedimentos de treinamento podem ser classificados de duas formas:

(a) aprendizagem supervisionada; e

(b) não supervisionada.

A primeira, conceitualmente, considera o aprendizado supervisionado por um "professor" que conhece um conjunto de exemplos de entradas-saídas.

O processo de treinamento funciona da seguinte maneira: aplica-se o vetor de entrada, a saída da rede é calculada e comparada com o vetor-alvo correspondente. Encontra-se um erro, que é realimentado através da RNA, e os pesos são atualizados de acordo com o algoritmo escolhido com o objetivo de minimizar esse erro. Repete-se o processo até que o erro para os vetores de treinamento alcance níveis ínfimos (BOCANEGRA, 2002). Essa forma de aprendizagem descrita é a forma de aprendizagem por correção do erro; é nessa regra de aprendizagem que se baseia o algoritmo da retropropagação do erro (ou error backpropagation), a ser utilizado neste trabalho e, portanto, explanado no próximo item.

Haykin (2001) subdivide o processo de aprendizagem sem professor em duas categorias:

(a) aprendizagem por esforço; e

(b) aprendizagem não supervisionada.

Segundo Pizarro (2005), nesse tipo de aprendizagem, não há exemplos rotulados da função a ser aprendida pela rede.

\section{Algoritmo da retropropagação ou backpropagation}

$\mathrm{O}$ algoritmo da retropropagação consiste em dois passos através de diferentes camadas da RNA, a propagação (passo para frente); e a retropropagação (passo para trás). Haykin (2001) descreve a propagação como um padrão de atividade (vetor de entrada) que é aplicado aos nós sensoriais da rede, e seu efeito propaga-se através da rede camada por camada, produzindo um conjunto de saídas como resposta real da rede. Nesse passo, os pesos sinápticos da RNA são todos fixos. Já durante a retropropagação, ocorre o ajuste dos pesos de acordo com a regra da correção do erro. Em outras palavras, a resposta real da rede é 
subtraída da resposta-alvo, produzindo um sinal de erro. Este, por sua vez, é propagado através da RNA na direção inversa das conexões sinápticas (daí a nomenclatura "retropropagação do erro"). Para que a resposta real aproxime-se da respostaalvo em um sentido estatístico, os pesos sinápticos são ajustados.

\section{Método}

\section{Aquisição dos dados para a regressão}

Os dados utilizados para a análise das regressões foram obtidos em pesquisa anteriormente realizada, na qual salas de escritório foram modeladas e simuladas. O conjunto de dados corresponde a 216 modelos, divididos em três geometrias diferentes, onde apenas a profundidade dos ambientes possui diferentes tamanhos (ver Figura 1) (DIDONÉ, 2009). Mais detalhes dessa pesquisa estão apresentados a seguir.

O consumo de energia dessas tipologias foi obtido através da simulação computacional, com a integração entre dois programas: o Daysim/Radiance e o EnergyPlus. O Daysim serviu para avaliar o comportamento dinâmico da luz natural e obter os dados energéticos necessários para a simulação energética integrada no EnergyPlus, que foi utilizado para a obtenção dos dados referentes ao consumo energético necessários no desenvolvimento da correlação numérica.

Para os dados de entrada no programa de simulação foram utilizadas as características construtivas, de uso e ocupação dos modelos, segundo a Tabela 1 e o relatório do controle de iluminação artificial obtido na simulação de iluminação.

As refletâncias internas utilizadas foram:
(a) 70\% para o teto;
(b) $50 \%$ para as paredes; e
(c) $20 \%$ para o piso.

Figura 1 - Esquema da geometria dos modelos Fonte: Didoné (2009).

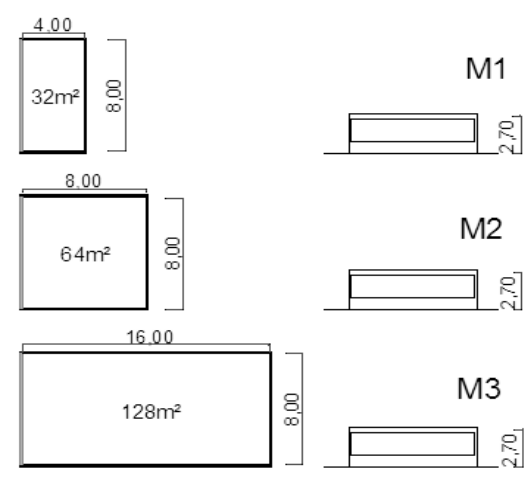

Tabela 1 - Características construtivas, de uso e ocupação e de iluminação

\begin{tabular}{|c|c|c|}
\hline \multirow{2}{*}{ Transmitância térmica $\left(\mathrm{W} / \mathrm{m}^{2} \mathrm{~K}\right)$} & Paredes & 2,47 \\
\hline & Cobertura & 2,42 \\
\hline \multirow{2}{*}{ Capacidade térmica $\left[\mathrm{KJ} /\left(\mathrm{m}^{2} . \mathrm{K}\right)\right]$} & Paredes & 200 \\
\hline & Cobertura & 187 \\
\hline \multirow{2}{*}{ Absortância } & Paredes & 0,65 \\
\hline & Cobertura & 0,70 \\
\hline \multicolumn{3}{|c|}{ Ocupação média (m²/pessoa) } \\
\hline \multirow{2}{*}{ Densidade de carga interna $\left(\mathrm{W} / \mathrm{m}^{2}\right)$} & Iluminação & 7 \\
\hline & Equipamentos & 9,7 \\
\hline \multirow{3}{*}{ Padrão de uso (horas) } & Ocupação & $8-18$ \\
\hline & Iluminação & $8-18$ \\
\hline & Equipamentos & 8-18 \\
\hline \multirow{3}{*}{$\begin{array}{l}\text { Características do sistema de } \\
\text { condicionamento de ar }\end{array}$} & Tipo & Aparelho de janela \\
\hline & Capacidade de refrigeração (BTU/h) & Ideal para o protótipo \\
\hline & COP (Wtérmico/Welétrico) & 3,15 \\
\hline
\end{tabular}

Fonte: adaptado de Santana (2006) e Carlo (2008). 
Para o sistema de iluminação artificial foi definido um projeto eficiente com luminárias de embutir de alta eficiência e aletas metálicas que impedem o ofuscamento. Foram utilizadas lâmpadas fluorescentes tubulares T5 de 28 W. A iluminância de projeto seguiu as recomendações da NBR 5413 (ABNT, 1992), com valor de 500 lux. Como resultado final da análise tem-se o consumo energético em $\mathrm{kWh} / \mathrm{m}^{2}$.

Os parâmetros simulados e as convenções para a análise de Regressão Linear Multivariada são apresentados na Tebela 2. Na tabela, as colunas com "S" correspondem aos valores utilizados nos modelos simulados, e as colunas com "E" correspondem aos valores convertidos utilizados na equação. Como descrito na tabela, os valores utilizados na equação devem ser normalizados entre 0 e 1 . Para a variável PROF (profundidade) utiliza-se uma razão do valor da profundidade do ambiente por $20(\mathrm{PROF} / 20)$, já que para a equação em desenvolvimento é possível avaliar ambientes com profundidade de até $20 \mathrm{~m}$.

\section{Regressão Linear Multivariada}

A análise com Regressão Linear Multivariada foi escolhida para a avaliação de diferentes modelos de edificações, a fim de elaborar um indicador de consumo de eficiência energética com o uso da iluminação natural. Os dados utilizados neste trabalho foram obtidos na pesquisa de Didoné (2009) e encontram-se descritos no item anterior.

O modelo de regressão foi elaborado com o software SPSS $16.0^{5}$. Os dados de consumo, obtidos através de simulação computacional, foram analisados pelo programa, e uma equação que relaciona as variáveis em estudo foi gerada. As variáveis são a orientação, a área de abertura, o fator solar do vidro, os ângulos verticais e horizontais de sombreamento, e a profundidade do ambiente.

O programa forneceu as estatísticas de uma reta usando o método dos mínimos quadrados, para obter uma equação linear que ajuste os dados, retornando uma matriz que a descreve. A relação que descreve o modelo é (Equação 1):

$\mathrm{y}=\beta_{0}+\beta_{1} \mathrm{X}_{1}+\beta_{2} \mathrm{X}_{2}+\ldots+\beta_{\mathrm{n}} \mathrm{X}_{\mathrm{n}}$.

Os valores de $\beta$ são coeficientes que correspondem a cada valor de $X$. A tendência linear de $y$ das seis variáveis $X$ inseridas pode ser descrita como:

(a) o $y$ corresponde ao consumo de energia elétrica;

\footnotetext{
${ }^{5}$ SPSS 16.0 é um programa computacional com um sistema para análise de dados que permite desenvolver um indicador de consumo (IC) para avaliar a eficiência energética da edificação com a influência da luz natural.
}

(b) o $X$ corresponde às variáveis consideradas relevantes para o aproveitamento da luz natural no desempenho energético de edificações; e

(c) o $n$ corresponde ao número de variáveis $X$ utilizadas na equação.

Após a obtenção da equação, algumas análises foram necessárias. Essas análises incluíram o coeficiente de determinação ou de explicação $\left(\mathrm{r}^{2}\right)$, o desvio padrão para a estimativa de y e o teste $\mathrm{t}(t$ Student), que serviu para testar a igualdade das médias do consumo utilizado (simulado) com as médias dos consumos obtidos pela equação.

Para atingir o objetivo proposto, os mesmos dados de entrada para a análise através da Regressão Linear Multivariada foram utilizados como dados de entrada para a análise através das RNAs, tornando possível a comparação entre os dois modelos de análise de regressão, para representar o potencial de economia de energia com o uso da iluminação natural.

\section{Redes Neurais Artificiais}

A Regressão Não Linear Multivariada deu-se através de RNA; esta foi treinada e testada com o programa Matlab. Para a criação de uma rede neural foram adotados os seguintes passos:
(a) definição dos padrões;
(b) inicialização da rede;
(c) definição dos parâmetros de treinamento;
(d) treinamento da rede; e
(e) teste da rede.

Como primeiro passo, os dados da simulação integrada foram formatados de maneira que os parâmetros de entrada pudessem ser lidos pela RNA, bem como os dados de saída. Para tal, os parâmetros de entrada foram tratados de duas maneiras: os que correspondiam às variáveis qualitativas foram tratados de forma binária, sendo indicado o número 1 quando a variável estava presente, e 0 para quando não estava presente. Já os parâmetros representados por variáveis quantitativas contínuas foram normalizados. O tipo da normalização depende da função de transferência (de neurônio para neurônio, aplicada ao $\left.n e t^{6}\right)$ que será utilizada.

168 Fonseca, R. W. da; Didoné, E. L.; Pereira, F. O. R.

\footnotetext{
${ }^{6}$ Net: sinal total que entra no corpo de processamento do neurônio artificial. Seu valor é calculado através da multiplicação do sinal que chega ao neurônio pelo peso da sinapse em questão.
} 
Tabela 2 - Dados utilizados para regressão linear multivariada

\begin{tabular}{c|c|c|c|c|c|c|c|c|c|c|c}
\hline \multicolumn{2}{c|}{ Profundidade } & \multicolumn{2}{|c|}{ Orientação } & \multicolumn{2}{|c|}{ *FS } & \multicolumn{2}{c|}{$*$ PAF } & \multicolumn{2}{c}{ *AVS } & \multicolumn{2}{c}{ *AHS } \\
\hline S & E & S & E & S & E & S & E & S & E & S & E \\
\hline \multirow{2}{*}{$4 \mathrm{~m}$} & 0,2 & $0^{\circ}$ & 0 & & & $25 \%$ & 0,25 & & & & \\
$8 \mathrm{~m}$ & 0,4 & $90^{\circ}$ & 0,25 & 0,23 & 0,23 & $50 \%$ & 0,50 & $0^{\circ}$ & 0,00 & $0^{\circ}$ & 0,00 \\
$16 \mathrm{~m}$ & 0,8 & $180^{\circ}$ & 0,50 & 0,82 & 0,82 & $75 \%$ & 0,75 & $45^{\circ}$ & 0,50 & $45^{\circ}$ & 0,50 \\
\hline
\end{tabular}

Fonte: Didoné (2009).

Nota: Legenda:

*FS = fator solar do vidro;

*PAF = percentual de abertura da fachada;

*AVS = ângulo vertical de sombreamento; $e$

*AHS = ângulo horizontal de sombreamento.

No caso deste estudo, as variáveis quantitativas contínuas são profundidade, orientação, fator solar e PAF, enquanto as qualitativas são proteções solares AVS e AHS. Os ângulos de sombreamento foram considerados variáveis qualitativas, pois foi considerada sua existência ou não no modelo, sendo seu valor constante e igual a $45^{\circ}$.

Para a normalização, recomenda-se que sejam feitos dois grupos, a normalização entre 0 e 1, e entre -1 e 1 . Assim, diferentes tipos de funções podem ser testados durante o treinamento $\mathrm{e}$ validação da rede.

Como segundo passo, a inicialização da rede, definiu-se o tipo de rede. No caso deste estudo, adotou-se a Multilayer Perceptron MLP (feedfoward ou rede alimentada adiante). Definiuse o número de neurônios na camada oculta (camada intermediária entre as camadas de entrada e de saída), que foram determinados através de testes, seguidos das funções de transferência e treinamento. Existem várias funções de transferências e de treinamento, entretanto foram selecionadas duas de transferência e quatro de treinamento como as mais adequadas a este trabalho. As arquiteturas de rede foram inicialmente testadas com as funções de transferência $\operatorname{logsig}^{7}$ e tansig $^{8}$, e com a função de treinamento traingda ${ }^{9}$. Assim, as configurações que apresentaram maior coeficiente de correlação e

\footnotetext{
${ }^{7}$ Logsig: função de transferência logarítmica sigmoidal. Calcula o valor da saída do neurônio a partir das entradas do net, ou seja, o valor que será transferido para a próxima camada utilizando uma função logarítmica sigmoidal que varia entre 0 e +1 . Possui formato de " $S$ ", sendo uma função não linear.

${ }^{8}$ Tansig: função de transferência tangente hiperbólica sigmoidal, Apresenta a mesma definição da função anterior, entretanto utiliza como função de transferência a tangente hiperbólica sigmoidal, que varia entre -1 e +1 .

${ }^{9}$ Traingda: função de treinamento gradiente descendente com taxa de aprendizado adaptativo backpropagation. Essa função de treinamento atualiza os valores dos pesos e dos baias de acordo com o gradiente descendente com taxa de aprendizado adaptativo. Os baias são uma unidade especial utilizada na camada de entrada, com a finalidade de aumentar os graus de liberdade, permitindo melhor adaptação, por parte da rede neural, ao conhecimento a ela fornecido.
}

menor erro quadrático médio (EQM) foram escolhidas.

Como parâmetros de treinamento foram definidos o gradiente mínimo ${ }^{10}$, o número máximo de épocas ${ }^{11}$ que a rede foi treinada, o erro final desejado e a taxa de aprendizado ${ }^{12}$.

Para o treinamento e validação da RNAs foi utilizado o método da validação cruzada (ou cross validation). Esse método foi adotado por se tratar de um conjunto de dados com um número relativamente pequeno de casos para o treinamento de RNAs (multifold-cross validation).

Nesse método o conjunto de dados é dividido em dois subconjuntos. O primeiro é o subconjunto de treinamento, são os exemplos apresentados à rede. E o segundo é o subconjunto teste. O conjunto teste tem de $10 \%$ a $20 \%$ do conjunto de treinamento; no caso deste trabalho foram adotados $10 \%$.

$\mathrm{O}$ conjunto de dados original de $\mathrm{N}$ exemplos é então dividido em $\mathrm{K}$ subconjuntos, onde $\mathrm{K}>1$. $\mathrm{O}$ treinamento do modelo ocorre em todos os subconjuntos exceto em um, e o erro de validação é medido testando-o sobre o subconjunto deixado de fora. $\mathrm{O}$ procedimento descrito é repetido por $\mathrm{K}$ tentativas, cada vez utilizando um subconjunto de validação diferente (HAYKIN, 2001). A 0ilustra o procedimento de um conjunto com $\mathrm{K}=3$ tentativas.
${ }^{10}$ Gradiente mínimo de descida: determina um vetor de pesos que minimiza o erro, o parâmetro gradiente mínimo estabelece o desempenho mínimo desse gradiente.

11 Épocas: número de interações para o treinamento.

12 Taxa de aprendizado, que geralmente varia entre 0,1 e 1.

Quanto mais baixo for seu valor, mais lento será o aprendizado. 


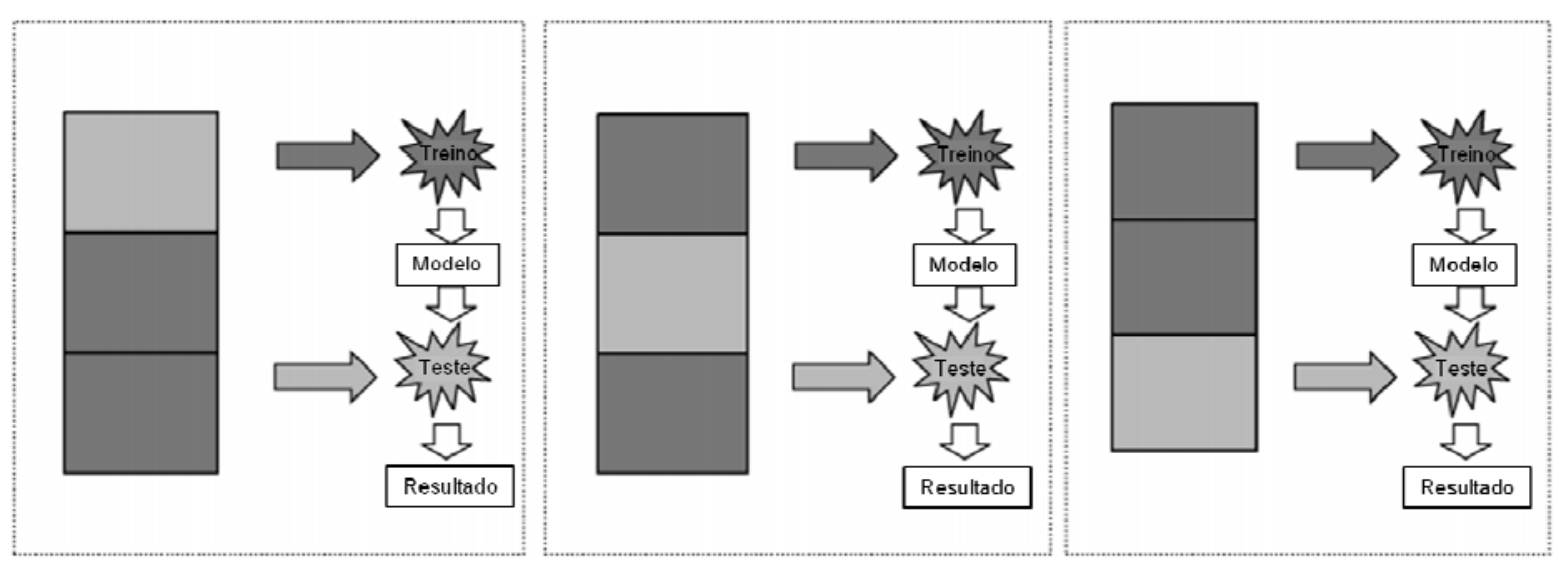

Figura 2 - Validação cruzada com K=3 tentativas

Fonte: adaptado de Refaeilzadeh, Tang e Liu (2008).

Essa seleção de dados é feita de forma randômica. Para o presente trabalho foram realizadas $\mathrm{K}=10$ simulações no programa Matlab, de onde foram obtidos o coeficiente de correlação de cada uma das configurações do subconjunto de treinamento, o erro absoluto (Eabs) e o erro quadrático médio (EQM) de cada subconjunto de validação. Com isso, foi possível averiguar a capacidade da rede em predizer resultados de saída.

Essa seleção de dados é feita de forma randômica. Para o presente trabalho foram realizadas $\mathrm{K}=10$ simulações no programa Matlab, de onde foram obtidos o coeficiente de correlação de cada uma das configurações do subconjunto de treinamento, o erro absoluto (Eabs) e o erro quadrático médio (EQM) de cada subconjunto de validação. Com isso, foi possível averiguar a capacidade da rede em predizer resultados de saída.

Para a determinação da arquitetura da rede, o número de neurônios da camada oculta, bem como as funções de transferência e treinamento são de grande importância. Segundo Costa (2001), como em geral o número escolhido de camadas ocultas e de neurônios que irão compô-las ocorre por experimentação, torna-se necessário testar redes com diversas configurações. Cada configuração de rede deve ser testada e, para cada caso, um valor de erro é calculado. Neste caso será o erro quadrático médio (EQM), obtido pela Equação 2.

$B Q M=\frac{\sum(0-e)^{2}}{n}$

Eq. 2

Onde:

o: representa o valor observado;

e: o valor estimado pelo modelo; e

n: é igual ao número de registros.

Assim, são gerados valores de erro para cada tipologia de rede. A tipologia que apresentar valor mínimo para EQM será a tipologia escolhida, ou seja, a que fornece estimativas mais próximas aos valores observados.

\section{Resultados}

\section{Equação Linear Multivariada}

Algumas etapas foram seguidas para a obtenção da equação de regressão multivariada. Inicialmente, foi realizado um estudo com o programa SPSS 16.0 para a elaboração de uma equação com as seis variáveis em estudo. Nessa etapa inicial, a variável orientação foi excluída pelo programa por não contribuir significativamente para o modelo, já que seus valores não foram relevantes na análise do consumo de energia elétrica.

Nesse estudo inicial, a correlação dos dados do consumo equacionado (referente aos valores obtidos com o uso da Equação 1) com os dados do consumo simulado (referente aos valores obtidos com a simulação computacional integrada) resultou na Equação 3, com um coeficiente de correlação (r) de 0,70 e um coeficiente de determinação $\left(\mathrm{r}^{2}\right)$ de 0,4922 .

ICRLuzNatR $=82,39+15,282 \times$ PAF $-11,18 \times$ AVS - 8,799 x AHS + 5,303 x FS - 6,1 x PROF

Eq. 3

Onde:

ICLuzNat: indicador de consumo com o uso da luz natural $\left[\mathrm{kWh} / \mathrm{m}^{2} / \mathrm{ano}\right]$;

PAF: porcentagem de abertura na fachada [\%];

AVS: ângulo vertical de sombreamento [grau];

AHS: ângulo horizontal de sombreamento [grau];

FS: fator solar [\%]; e

PROF: profundidade [razão (PROF/20)]. 


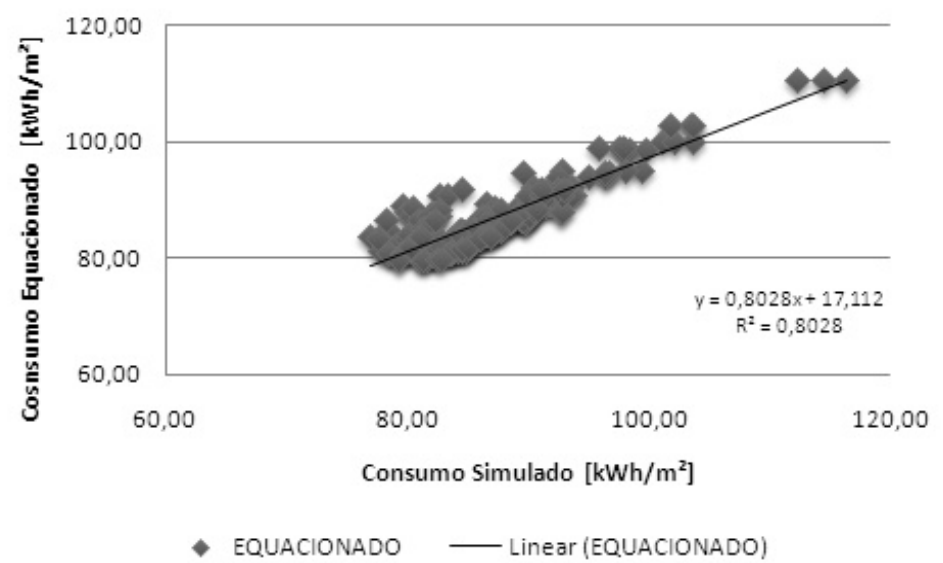

Figura 3 - Consumo anual de energia Simulado X Equacionado para edificações

Após avaliar a influência das variáveis utilizadas individualmente na Equação 3, optou-se por associá-las com o intuito de elaborar uma equação que apresentasse um resultado mais satisfatório (Equação 4). As associações foram realizadas mediante divisões e/ou multiplicações entre as variáveis, de acordo com suas relações e desempenho apresentado. As associações que apresentaram o resultado mais satisfatório foram: PAF/PROF; FSxPAF, PAFxAVSxFS/PROF, PAFxAHSxFS/PROF, PAFxAVSxFS e PAFxAHSxFS. Como resultado obteve-se um coeficiente de correlação linear (r) de 0,79 e um coeficiente de determinação $\left(\mathrm{r}^{2}\right)$ de 0,6243.

$\mathrm{ICLuzNat}=79.033+3,347 \times \mathrm{PAF} / \mathrm{PROF}+$ $23,109 \times$ FS $\times$ PAF $-7,433 \times$ PAF $\times$ AVS $x$ FS/PROF - 5,34 x PAF x AHS x FS/PROF 20,903 x PAF x AVS x FS - 18,20 x PAF x AHS x $\mathrm{FS}$ Eq. 4

Esses coeficientes são melhorados ao adotar 95\% dos valores em torno da reta de regressão, considerando esse valor como o nível de confiança $^{13}$ adequado para realizar a análise dos dados. Após o ajuste, os coeficientes de correlação (r) e de determinação $\left(\mathrm{r}^{2}\right)$ foram respectivamente 0,89 e 0,8028 , sendo a equação final resultante obtida a partir de 205 casos simulados, que se encontravam no nível de confiança determinado pela reta de regressão (ver Equação 5).

\footnotetext{
${ }^{13}$ Intervalos de confiança são construídos para indicar que, em $x \%$ das vezes que essa experiência for repetida, aquele valor seja detectado no estudo. São valores arbitrados segundo a ótica do pesquisador para atribuir alto grau de confiabilidade ao trabalho. Os valores usuais partem de $80 \%, 90 \%, 95 \%, 97,5 \%$ $99 \% 99,5 \%$ ou $99,9 \%$ É de bom senso utilizar um valor intermediário que permita analisar o grau de confiabilidade coerente com a realidade (MONTGOMERY; RUNGER, 2003).
}

$\mathrm{ICLuzNat}=78,090+4,187 \times \mathrm{PAF} / \mathrm{PROF}+$ $27,779 \times$ FS x PAF - 9,510 x PAF x AVS $x$ FS/PROF - 4,384 x PAF x AHS x FS/PROF $26,188 \times$ PAF $x$ AVS x FS - 28,795 x PAF x AHS $x$ FS

Os dados da equação final resultaram em um desvio padrão de 2,90 , e o teste $t$ mostrou que as médias das amostras simuladas e equacionadas são consideradas iguais. Utilizando-se as variâncias referentes a cada coeficiente adotado na equação, o t calculado foi de 90,85 (ver Figura 3).

\section{Redes Neurais Artificiais}

Como explanado no capítulo de metodologia, iniciou-se o processo de aproximação de funções por RNA pela normalização dos dados obtidos. A normalização foi feita primeiramente objetivando dados entre 0 e 1 , e posteriormente entre -1 e +1 . As duas normalizações foram feitas a fim de permitir testar o desempenho da rede com duas funções de transferência logsig (logarítmica) e tansig (tangente hiperbólica) respectivamente.

Como segundo passo foram criadas as planilhas do Matlab com os dados de entrada e de saída, que foram separadas em parâmetros de entrada e parâmetro de saída ou alvo.

Foram criadas, então, as planilhas para armazenar os resultados dos conjuntos de teste ou validação da rede. As planilhas criadas foram atual (ou dados originais), prediction (ou dados preditos pela rede), abserr (erro absoluto de cada caso de teste), squerr (EQM de cada caso de teste), mae (erro absoluto do conjunto de teste) e rmse (EQM do conjunto de teste). Esse conjunto de planilhas foi criado para cada um dos 10 testes da validação cruzada (cross validation).

Para a aplicação da validação cruzada (cross validation), o conjunto de dados referentes aos 
modelos simulados por Didoné (2009) foi dividido em 10 subconjuntos $\mathrm{K}$, e o conjunto de validação foi considerado 1/K. Assim, o conjunto com 216 casos foi subdividido em subconjunto de treinamento, com 195 casos, e subconjunto de teste ou validação, com 21 casos.

Para a inicialização da rede prepararam-se os conjuntos de treinamento e teste. A rede foi testada com as funções de transferência (função de ativação) logsig e tansig, e os resultados obtidos foram bastante próximos. $\mathrm{O}$ teste foi feito de forma completa, com as 10 rodadas da validação cruzada. A Tabela 3 traz os resultados de coeficiente de determinação e correlação, EQM e Eabs para os conjuntos utilizando ambas as funções. Como se pode notar, os coeficientes de correlação e determinação obtidos através das duas funções são muito próximos; se arredondados para duas casas decimais, ficariam iguais. Já os valores de EQM e Eabs do grupo da função logsig apresentaram resultados mais baixos. Esse foi o argumento de decisão para a escolha da função logsig para a rede.

Quanto à função de treinamento (ou algoritmo de treinamento), adotou-se a função traingda. Com essa função os resultados obtidos já foram bastante satisfatórios, então se julgou dispensável o treino com outras funções.

A arquitetura da rede ficou estruturada com seis parâmetros de entrada, dez neurônios na camada oculta e um parâmetro de saída. Os números de parâmetros de entrada e saída não variam, visto que correspondem respectivamente às variáveis dos modelos e ao consumo de cada um. Já o número de neurônios na camada oculta pode variar, entretanto através dos testes realizados, dez neurônios mostrou-se um número adequado.

Como parâmetros de treinamento foram definidos o gradiente mínimo como 0,0001 ; o número de máximo de épocas que a rede será treinada como 10.0000; o erro final desejado como 0,000001; e a taxa de aprendizado, 0,001.

Para a adoção do número de épocas no treinamento, iniciou-se com 10.000 épocas, passando a 20.000, 50.000 e, por fim, 100.000 épocas. Apesar de demandar um pouco mais de tempo de treinamento, julgou-se este um número adequado de épocas de treinamento para esse problema. A Figura 4 ilustra o gráfico do Matlab, que traz a época em que ocorreu o melhor desempenho de treinamento da rede. $\mathrm{Na}$ maior parte dos casos, o melhor desempenho ficou na casa das 99.900 épocas.

A Figura 5 ilustra o gráfico de teste ou validação para as 10 rodadas da validação cruzada para função logsig. Nos gráficos de teste ou validação são ilustrados os valores reais mostrados à rede, chamados nos gráficos de "atual" e representados pela cor cinza-claro. Já em cinza escuro são os valores preditos pela rede para aquele conjunto. Em todos os casos pode-se observar uma representatividade significativa dos dados, sendo a rede considerada bastante adequada para o problema. O desvio padrão de todo o conjunto foi considerado baixo, igual a 0,1565 .

Pode-se notar que a rede representou muito bem os resultados reais. Como confirmação, foram apresentados na Tabela 3 os dados de $\mathrm{r}^{2}$ e r de cada conjunto treinado e erro absoluto (Eabs) e EQM dos conjuntos de teste. Pode-se perceber que em todos os casos de treinamento o $\mathrm{r}^{2}$ foi superior a 0,99 , o que representa uma boa aproximação dos dados.

O EQM dos conjuntos de testes variou entre $0,02 \mathrm{e}$ 0,05 , e a média ficou em 0,0303 , inferior a 0,05 , como recomenda a bibliografia. Já o Eabs não ultrapassou 0,05 em nenhum dos casos.

\section{Tabela 3 - Comparativo entre resultados obtidos através das funções de transferência logsig e tansig}

\begin{tabular}{c|c|c|c|c|c|c|c|c}
\hline \multirow{2}{*}{ TESTE } & \multicolumn{3}{|c|}{ Grupo Função Logsig } & \multicolumn{3}{c}{ Grupo Função Tansig } \\
\cline { 2 - 10 } & $\mathbf{r}^{\mathbf{2}}$ & $\mathbf{r}$ & EQM & Eabsol & $\mathbf{r}^{\mathbf{2}}$ & $\mathbf{r}$ & EQM & Eabsol \\
\hline 1 & 0,9897 & 0,9949 & 0,0225 & 0,0174 & 0,9895 & 0,9947 & 0,0409 & 0,0334 \\
\hline 2 & 0,9882 & 0,9941 & 0,0335 & 0,0246 & 0,9902 & 0,9951 & 0,0331 & 0,0218 \\
\hline 3 & 0,9873 & 0,9937 & 0,0300 & 0,0214 & 0,9905 & 0,9953 & 0,0528 & 0,0388 \\
\hline 4 & 0,9867 & 0,9933 & 0,0277 & 0,0233 & 0,9885 & 0,9942 & 0,0642 & 0,0475 \\
\hline 5 & 0,9877 & 0,9938 & 0,0195 & 0,0139 & 0,9866 & 0,9933 & 0,0587 & 0,0394 \\
\hline 6 & 0,9829 & 0,9914 & 0,0326 & 0,0255 & 0,9891 & 0,9945 & 0,0663 & 0,0513 \\
\hline 7 & 0,9885 & 0,9942 & 0,0210 & 0,0172 & 0,9907 & 0,9953 & 0,0480 & 0,0358 \\
\hline 8 & 0,9900 & 0,9950 & 0,0348 & 0,0182 & 0,9924 & 0,9962 & 0,0695 & 0,0435 \\
\hline 9 & 0,9821 & 0,9910 & 0,0532 & 0,0362 & 0,9902 & 0,9951 & 0,0329 & 0,0271 \\
\hline 10 & 0,9838 & 0,9919 & 0,0280 & 0,0236 & 0,9920 & 0,9960 & 0,0845 & 0,0508 \\
\hline MÉyDIA & 0,9867 & 0,9933 & 0,0303 & 0,0221 & 0,9900 & 0,9950 & 0,0551 & 0,0389 \\
\hline
\end{tabular}

172 Fonseca, R. W. da; Didoné, E. L.; Pereira, F. O. R. 


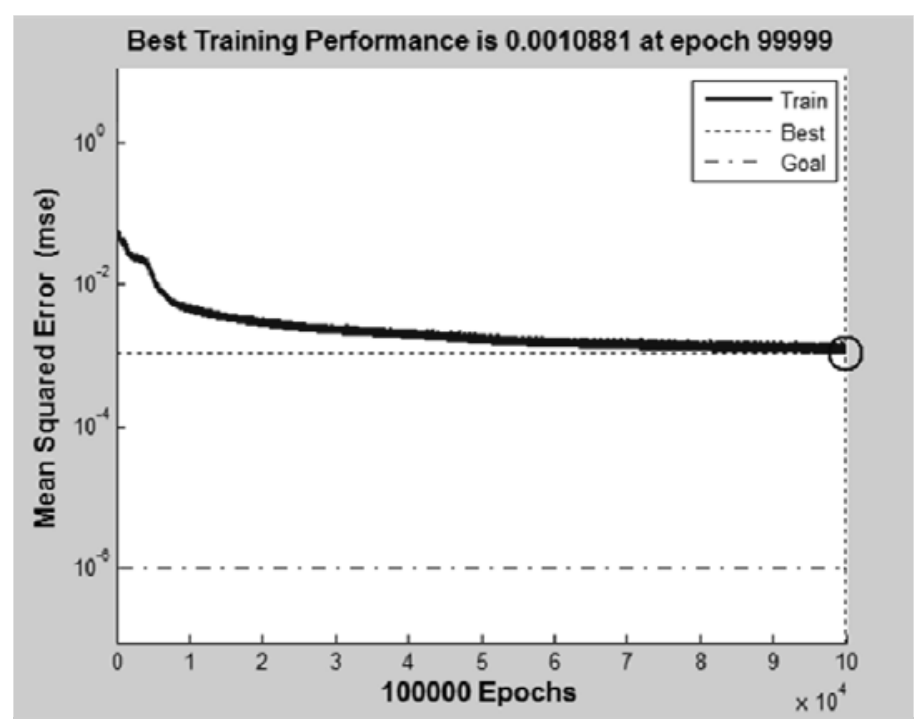

Figura 4 - Gráfico do Matlab ilustrando a época em que ocorreu o melhor desempenho do treinamento

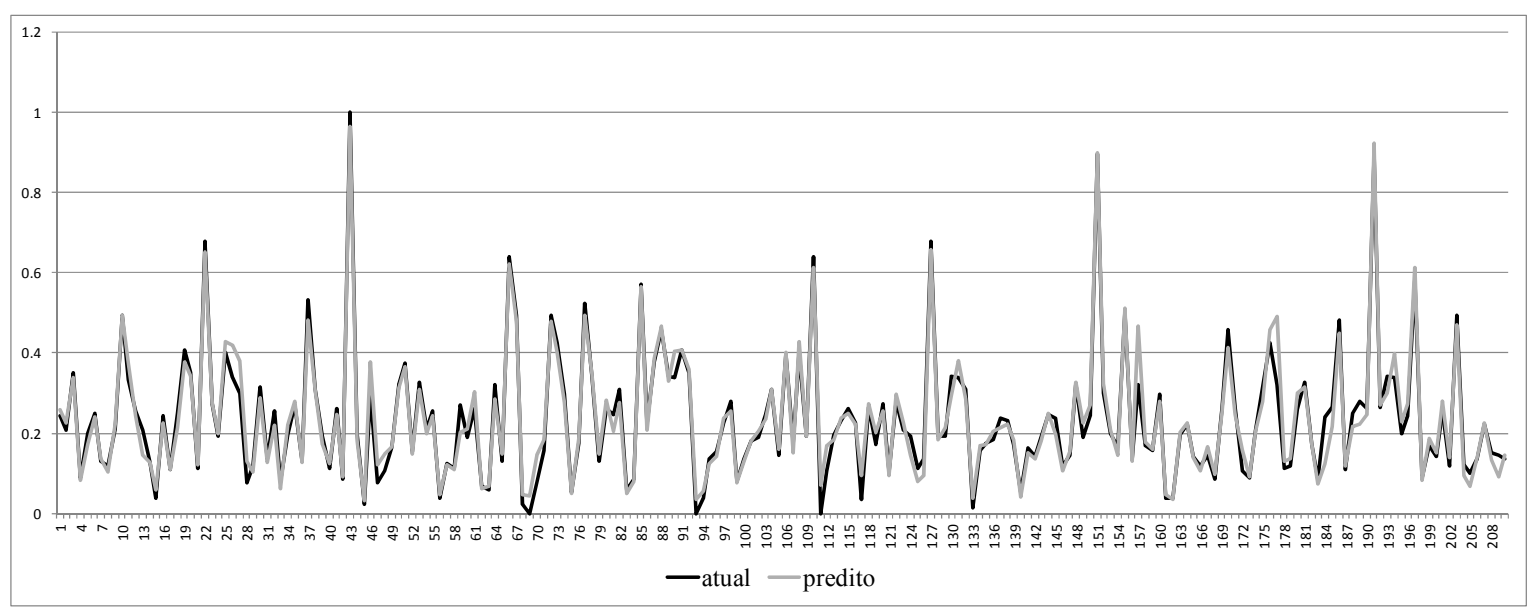

Figura 5 - Gráfico comparativo entre valores reais e preditos pela rede do grupo de validação

\section{Comparações entre os modelos de regressão}

A comparação entre os coeficientes de determinação obtidos pelas duas formas de regressão indicam que o modelo não linear representa melhor a função que descreve a questão do consumo energético considerando variáveis que influenciam na iluminação natural. $\mathrm{O} \mathrm{r}^{2}$ médio obtido pela RNA tanto para a função logsig quanto para tansig, quando arredondado para duas casas decimais, chegou a 0,99 , enquanto no melhor caso da Regressão Linear Multivariada chegou a um máximo de 0,8. Já o desvio padrão para a Regressão Linear Multivariada foi de 2,90, enquanto o da RNA foi de 0,1565 , demonstrando menor dispersão dos dados.

\section{Conclusões}

A simulação computacional energética integrada à simulação de iluminação natural mostrou-se uma ferramenta confiável para a predição do consumo energético em edificações. Permite de maneira satisfatória a predição do comportamento energético de edifícios por meio de estudos paramétricos para avaliação do efeito de cada variável no consumo final da edificação. Além disso, mostrou-se uma forma consistente para a aquisição de dados para a modelagem de regressões multivariadas.

Foram comparadas as Regressões Lineares Multivariadas e Não Lineares (RNAs). Comparando o $\mathrm{r}^{2}$ obtido utilizando a Regressão Linear Multivariada com o obtido utilizando RNAs, pode-se perceber uma melhora no coeficiente de determinação. Para a Regressão Linear Multivariada, quando considerado todo o 
conjunto de dados, obteve-se um $\mathrm{r}^{2}=0,6243$. Reduzindo esse conjunto, considerando apenas 95\% dos casos, obteve-se melhora, chegando a $\mathrm{r}^{2}=0,8028$. Já com a utilização de RNAs, considerando todo o conjunto de dados, o $\mathrm{r}^{2}$ médio das dez rodadas de validação foi de 0,9867 . Esses resultados confirmam a utilização de RNAs como uma boa alternativa à representação dos modelos no caso deste estudo.

Além disso, as RNAs apresentaram altos valores de coeficiente de correlação e determinação, bem como baixos EQM e Eabs. A média das dez validações do EQM para a função logsig foi de 0,0303, abaixo de 0,05 , como recomenda a literatura. Com base nos resultados obtidos, as Redes Neurais Artificiais mostraram-se uma alternativa interessante na abordagem da aproximação de funções. Por meio deste estudo comparativo confirmou-se o melhor desempenho da técnica de Regressão Não Linear Multivariada em comparação com a Linear Multivariada.

A Regressão Linear Multivariada apresentada neste artigo foi utilizada para a formulação de uma equação de índice de consumo de iluminação natural. Em face do melhor desempenho para aproximação de funções das RNAs, aponta-se para o potencial da utilização destas para a determinação de índices de consumo de iluminação natural. Depois de validada, a RNA pode oferecer resultados de saída para dados de entrada que ainda não lhe foram apresentados. Isso pode ser feito com o programa Matlab, utilizando apenas o modo progressivo (feed-foward) ou viabilizando a utilização da rede por meio de planilha eletrônica.

\section{Referências}

\section{ASSOCIAÇÃO BRASILEIRA DE NORMAS TÉCNICAS. NBR-5413: iluminância de interiores. Rio de Janeiro, 1992. 13 p.}

ASSAF, L. O.; PEREIRA, F. O. R. Perspectivas de la Eficiência Energética en la Iluminación: desafios para el desarrollo. In: ENCONTRO NACIONAL SOBRE CONFORTO NO AMBIENTE CONSTRUÍDO, 7.; CONFERENNCIA LATINO AMERICANA SOBRE CONFORTO E DESEMPENHO ENERGÉTICO NAS EDIFICAÇÕES, 3., Curitiba, 2003. Anais... Curitiba: ANTAC, 2003.

BRASIL. Lei n. 10295, de 17 de outubro de 2001, que dispõe sobre a Política Nacional de Conservação e Uso Racional de Energia. Diário Oficial da União, Brasília, 2001a. Disponível em: $<$ www.inmetro.gov.br/qualidade/lei10295.pdf $>$. Acesso em: 31 jan. 2012.
BOCANEGRA, C. W. R. Procedimento para Tornar Mais Efetivo o Uso das Redes Neurais Artificiais em Planejamento de Transportes. 97 f. São Carlos, 2002. Dissertação (Mestrado em Engenharia Civil) - Departamento de Engenharia Civil, Universidade de São Paulo, São Carlos, 2002.

CARLO, J. C. Desenvolvimento de Metodologia de Avaliação da Eficiência Energética da Envoltória de Edificações Não Residenciais. Florianópolis, 2008. Tese (Doutorado em Engenharia Civil) - Centro Tecnológico, Universidade Federal de Santa Catarina, Florianópolis, 2008.

CARLO, J. C.; GHISI, E.; LAMBERTS, R. Energy Efficiency Building Code of Salvador. In: INTERNATIONAL BUILDING PERFORMANCE SIMULATION ASSOCIATION CONFERENCE, 8., Eindhoven, 2003. Proceedings... Eindhoven: IBPSA, 2003a.

CARLO, J.; PEREIRA, F. O. R.; LAMBERTS, R. Iluminação Natural Para Redução do Consumo de Energia de Edificações de Escritório Aplicando Propostas de Eficiência Energética Para o Código de Obras do Recife. In: ENCONTRO NACIONAL DE TECNOLOGIA NO AMBIENTE CONSTRUíDO, 10., São Paulo, 2004. Anais... São Paulo, 2004.

COSTA, G. C. F. Uma Avaliação do Consumo de Energia Com Transportes em Cidades do Estado de São Paulo. 103 f. São Carlos, 2001. Dissertação (Mestrado em Engenharia Civil) Departamento de Engenharia Civil, Universidade de São Paulo, São Carlos, 2001.

DIDONÉ, E. L. A influência da Luz Natural na Avaliação da Eficiência Energética de Edifícios Contemporâneos de Escritórios em

Florianópolis/SC. 174 f. Florianópolis, 2009.

Dissertação (Mestrado em Arquitetura e

Urbanismo) - Universidade Federal de Santa Catarina, Florianópolis, 2009.

HAYKIN, S. Redes Neurais: princípios e práticas. Porto Alegre: ARTMED, 2001. 900 p.

KAZANASMAZ, T.; GÜNAYDIN, M.; BINOL, S. Artificial Neural Networks to Predict Daylight Illuminance in Office Buildings. Building an Environment, v. 44, n. 8, p. 1751-1757, ago. 2009.

LI, D. H. W.; TSANG, E. K. W. An Analysis of Daylighting Performance for Office Buildings in Hong Kong. Building and Environment, v. 43, n. 9, p. 1446-1458, sep. 2008. 
MAYHOUB, M. S.; CARTER, D. J. The Costs and Benefits of Using Daylight Guidance to Light Office Buildings. Building and Environment, v. 46, n. 3, p. 698-710, mar. 2011.

MONTGOMERY, D. C.; RUNGER, G. C. Estatística Aplicada e Probabilidade Para Engenheiros. 2. ed. Rio de Janeiro: Ed: LTC, 2003.

PIZARRO, P. R. Estudo de Variáveis do Conforto Térmico e Luminoso em Ambientes Escolares. 179 f. Bauru, 2005. Dissertação (Mestrado em Arquitetura) - Faculdade de Arquitetura, Artes e Comunicação, Universidade Estadual Paulista, Bauru, 2005.

PIZARRO, P. R.; SOUZA, L. C. L. Qualificação da Iluminação Natural em Interiores: aplicação das redes neurais artificiais e 3DSkyView. Ambiente Construído, v. 7, n. 1, p. 83-96, jan./mar. 2007.

REFAEILZADEH, P.; TANG, L.; LIU, H. CrossValidation. In: Manual. Publication Arizona State University, 2008. p. 1-6.

REINHART, C. F.; WIENOLD, J. The Daylighting Dashboard: a simulation-based design analysis for daylit spaces. Building and Environment, v. 46, n. 2, p. 386-396, 2011.

SANTANA, M. V. Influência de Parâmetros Construtivos no Consumo de Energia de Edifícios de Escritório Localizados em Florianópolis, SC. Florianópolis, 2006. Dissertação (Mestrado em Engenharia Civil) Pós-Graduação em Engenharia Civil, Universidade Federal de Santa Catarina, Florianópolis, 2006.
SIGNOR, R. Análise de Regressão do Consumo de Energia Elétrica Frente a Variáveis Arquitetônicas Para Edifícios Comerciais Climatizados em 14 Capitais Brasileiras. $122 \mathrm{f}$. Florianópolis, 1999. Dissertação (Mestrado em Engenharia Civil) - Curso de Pós-graduação em Engenharia Civil, Universidade Federal de Santa Catarina, Florianópolis, 1999.

SOUZA, M. B. Impacto da Luz Natural no Consumo de Energia Elétrica em Um Edifício de Escritórios em Florianópolis. $191 \mathrm{f}$. Florianópolis, 1995. Dissertação (Mestrado em Engenharia Civil) - Programa de Pós-Graduação em Engenharia Civil, Universidade Federal de Santa Catarina, Florianópolis, 1995.

SOUZA, M. B. D. Potencialidade de Aproveitamento da Luz Natural Através da Utilização de Sistemas Automáticos de Controle Para a Economia de Energia Elétrica. 208 f.

Florianópolis, 2003. Tese (Doutorado em Engenharia de Produção) - Programa de PósGraduação em Engenharia de Produção, Universidade Federal de Santa Catarina, Florianópolis, 2003.

VIANNA, N. S.; GONÇALVES, J. C. S. Iluminação e Arquitetura. São Paulo: Virtus, 2001. 378 p.

WESTPHAL, F.; LAMBERTS, R. Regression Analysis of Electric Energy Consumption of Commercial Buildings in Brazil. In: Proceedings of Building Simulation. 2007. Disponível em: $<$ http://www.ibpsa.org/proceedings/BS2007/p732 final.pdf $>$. Acesso em: 10 set. 2011.

Revista Ambiente Construído

Associação Nacional de Tecnologia do Ambiente Construído

Av. Osvaldo Aranha, 99 - 3o andar, Centro

Porto Alegre - RS - Brasil CEP 90035-190

Telefone: +55 (51) 3308-4084 Fax: +55 (51) 3308-4054

www. seer. ufrgs. br/ ambienteconstruido

E-mail: ambienteconstruido@ufrgs.br 